\title{
Wideband Dual-Element Antenna Array for MIMO Mobile Phone Applications
}

\author{
Yuanqiang Wang, Jie Yang, Shangfu Hao, and Xiao Zhang \\ School of Information Science and Engineering, Hebei North University, Hebei, Zhangjiakou 075000, China \\ Correspondence should be addressed to Shangfu Hao; 184172328@qq.com
}

Received 15 May 2015; Revised 7 July 2015; Accepted 8 July 2015

Academic Editor: Wei Liu

Copyright (C) 2015 Yuanqiang Wang et al. This is an open access article distributed under the Creative Commons Attribution License, which permits unrestricted use, distribution, and reproduction in any medium, provided the original work is properly cited.

A printed dual-element antenna array for LTE MIMO mobile phone applications is presented in this paper. The two array elements are symmetrically placed with the same dimensions, and each of the them consists of a novel driven strip and a wandering shorting strip. The driven strip is a whole loop, which improves the impedance matching for the upper band. Therefore, the bandwidth coverage is expanded and the antenna size is minimized at the same time. In addition, thanks to the protruded ground on the ground plane, the antenna isolation between the two array elements is significantly enhanced. The proposed planar antenna array successfully covers the seven operating bands of GSM850/900/1800/1900/UMTS2100/LTE2300/2500, and the isolation is more than $10 \mathrm{~dB}$. The prototype was fabricated and tested, with $S$ parameters, efficiency, radiation patterns, envelop correlation coefficient (ECC), and ergodic capacity presented. From the measured results, it is indicated that the antenna array has excellent and reliable performances when it is applied in MIMO applications.

\section{Introduction}

Antenna array is recognized as an important technology for MIMO systems to achieve high downlink data transmission rate and reliable link in LTE wireless communications [1-4]. For MIMO mobile phone applications, the antenna arrays have to be compacted in a quite limited space; then the antenna elements will be closely spaced. In addition, the LTE mobile phones also need to be compatible with the previous communication standards, such as GSM and WCDMA. Therefore, the designed antenna array for mobile phone applications should be not only of small size but also capable of multiband operating. What is more, owing to the ever shrinking clearance area left on the ground plane, together with the strong electromagnetic couplings brought by metal components, it is filled with challenges to design a compact antenna array with good isolation for LTE mobile phone applications, especially for wideband references.

In the previous studies, several valid methods have been reported to achieve good isolation, such as protruded ground [5-7], decoupling networks $[8,9]$, polarization decoupling [10], and parasitic elements [11]. Nevertheless, when the structures of the antenna arrays become complex, especially for closely spaced cases, the mutual couplings will be much more complicated. For compact MIMO mobile phone applications, the decoupling networks discussed in $[8,9]$ and polarization method referred to in $[10]$ are not so applicable, which are mainly due to the limited bandwidth coverage and confined space. In [11], a parasitic element is used to realize enhanced isolation, but the sizes of the parasitic elements are usually too large. Accordingly, it is not practical for compact mobile phone applications, especially for the lower band. Under such circumstances, the optimization process of the decoupling schemes becomes hard and the workload for antenna designers will be greatly increased. Therefore, the implementation of small-size antenna arrays, with simple structures and good isolation, is more desired in practical MIMO mobile phone applications.

In this paper, a planar antenna array with simple structure and good isolation is presented and studied. The antenna array consists of two symmetrically located antenna elements, which separately comprises a driven strip and a shorting strip. The protruded ground, which is also applied in [5-7], is situated between the two antenna elements and helps to 


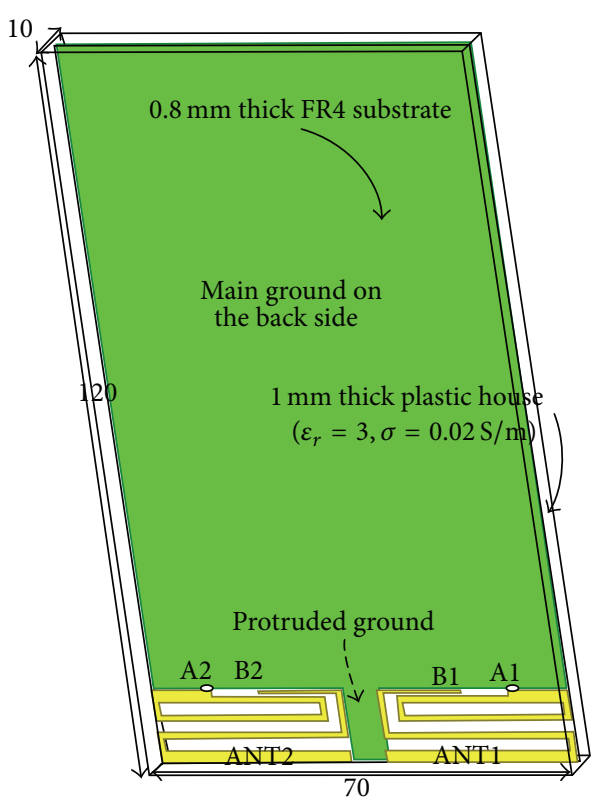

(a)

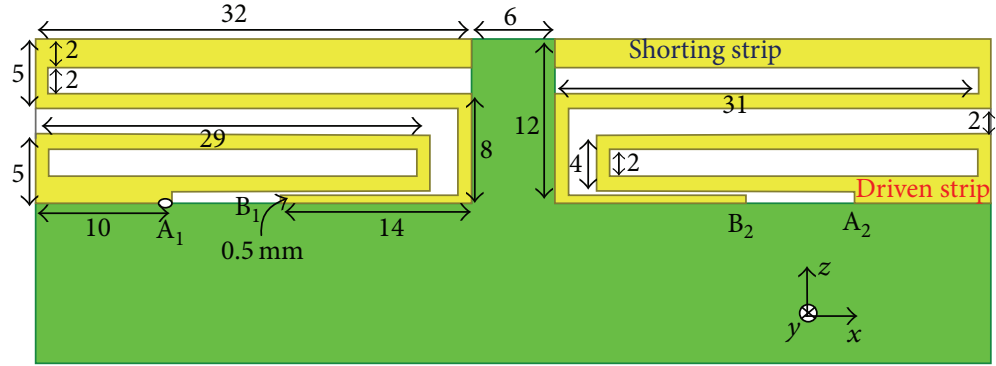

$A_{1}, A_{2}$ : feeding points

$\mathrm{B}_{2}, \mathrm{~B}_{2}$ : shorting points

(b)

FIgure 1: Proposed antenna array configuration. (a) Geometry of the proposed antenna array for mobile phone applications. (b) Detailed dimensions of the proposed antenna array. All dimensions are in millimeters.

reduce the mutual coupling between the two share-ground antenna elements. Compared with the previous designs with protruded ground [5-7], the proposed antenna array has a wider bandwidth yet with a smaller size; thus it is more valuable in practical MIMO mobile phone applications. The paper is organized in four sections. In Section 2, the antenna array topology and the relevant design considerations are presented, while the measured results are discussed in Section 3. Finally, in Section 4, some concluding remarks are reported.

\section{Proposed Antenna Array Design}

Figure 1(a) shows the geometry of the proposed printed antenna array for seven-band MIMO mobile phone applications. The array consists of two identical antenna elements and is symmetrically situated on the bottom of the system board. The system circuit board is served as a $0.8 \mathrm{~mm}$ FR4 substrate, with relative permittivity 4.4 and loss tangent 0.02 . On the back of the referred FR4 substrate, the system ground plane is divided into two parts, namely, the main ground plane of $70 \times 108 \mathrm{~mm}^{2}$ and the protruded ground plane of $6 \times 12 \mathrm{~mm}^{2}$. Two symmetrical no-ground regions are left to accommodate the proposed antenna array at the bottom of the circuit board. Figure 1(b) depicts the detailed dimensions and structure of the antenna array. To simulate the real surroundings of the practical mobile phones, a $1 \mathrm{~mm}$ thick plastic housing is employed, keeping a fixed distance of $0.5 \mathrm{~mm}$ to the edges of the FR4 substrate.

The shorting strip has a total length of about $86 \mathrm{~mm}$, which is capable of generating a 0.25 -wavelength resonant mode at about $880 \mathrm{MHz}$. Together with the effects of the loop driven strip, the operating bands of GSM850/900 are covered. For the upper band terms, the shorting strip is able to contribute a higher-order resonant mode at about $1800 \mathrm{MHz}$. With the similar principle, the driven strip introduces additional capacitive coupling and therefore shifts the existing resonant mode to higher frequency of about $2000 \mathrm{MHz}$, covering the GSM1800/1900/UMTS2100/LTE2300/2500 operation. To reduce the mutual coupling between the two antenna elements, the protruded ground is employed, which significantly improves the isolation between the two array elements to be more than $10 \mathrm{~dB}$. Finally, the proposed antenna array is able to cover the GSM850/900/1800/1900/UMTS2100/ LTE2300/2500 operation, with desired isolation of more than $10 \mathrm{~dB}$.

To investigate the working principles of the protruded ground, simulated surface current distributions at the frequencies of 925 and $2500 \mathrm{MHz}$ are plotted in Figures 2(a) and 2(b), respectively. The simulation is carried out under the condition that ANT1 is excited and ANT2 is terminated to a $50 \Omega$ matched load. For $925 \mathrm{MHz}$, as shown in Figure 2(a), the comparison of the surface current distributions with and without the protruded ground is quite significant. With the addition of the protruded ground, the current distributions on the ground plane are changed and the coupled current on the driven strip of ANT2 is visibly reduced, demonstrating that enhanced port isolation is achieved. For $2500 \mathrm{MHz}$, as shown in Figure 2(b), the addition of the protruded ground transforms the distributions of the current on the ground plane. Also, the coupled current on the port of ANT2 from ANT1 is reduced. Accordingly, the mutual coupling between the array elements is eliminated. This point is just in coincidence with the foregoing discussion. The mutual coupling for the whole desired band is below $-10 \mathrm{~dB}$, which is regarded as a design specification of the antenna arrays for MIMO mobile phone applications $[5,6]$. 

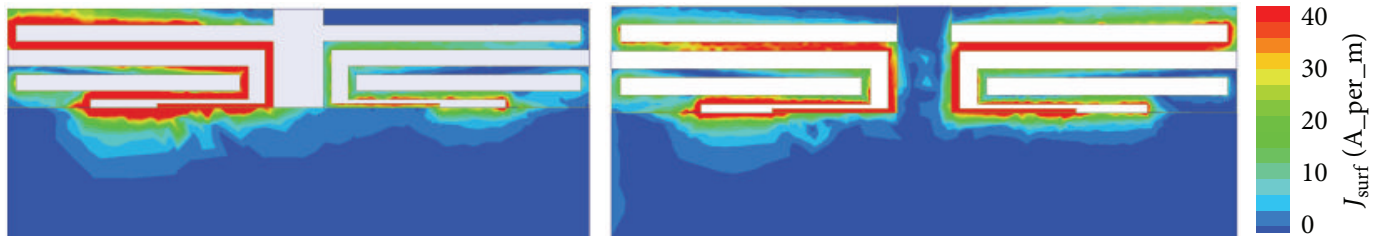

(a)
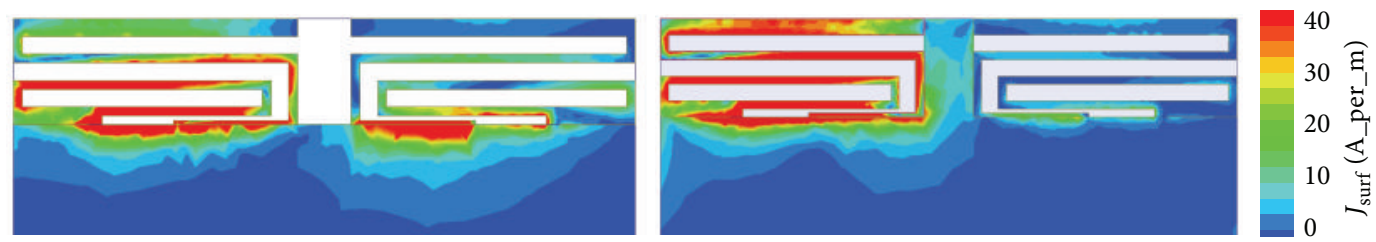

(b)

FIGURE 2: Simulated surface current distributions on the antenna elements and the system ground plane: (a) $925 \mathrm{MHz}$ with (right) and without (left) the protruded ground. (b) $2500 \mathrm{MHz}$ with (right) and without (left) the protruded ground.

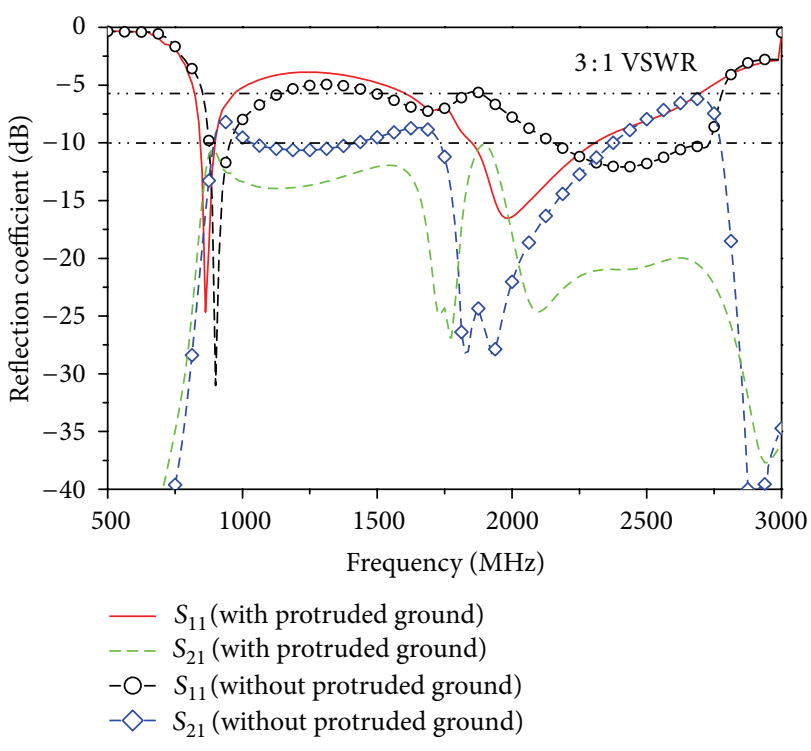

FIGURE 3: Simulated $S$ parameters with and without the protruded ground for the antenna array.

To better understand the effects of the protruded ground, simulated $S$ parameters of the antenna array with and without the protruded ground are shown in Figure 3. From Figure 3, it is seen that the addition of the protruded ground improves the impedance matching for the upper band. This is mainly because that the protruded ground brings new current paths on the ground plane, as illustrated above. For the lower band, the resonant mode is shifted to lower frequency, which is due to the additional inductive couplings between the shorting strip and the protruded ground. Through analyzing the changing trend of $S_{21}$, it is apparent that the protruded ground reduces the mutual coupling between the two antenna elements, both for the upper band and the lower band. Typically, at 925 and $2500 \mathrm{MHz}$, the mutual coupling has been reduced from $-8.1 \mathrm{~dB}$ and $-8 \mathrm{~dB}$ to $-12 \mathrm{~dB}$ and $-20 \mathrm{~dB}$ with the effects of the protruded ground, respectively. Therefore,

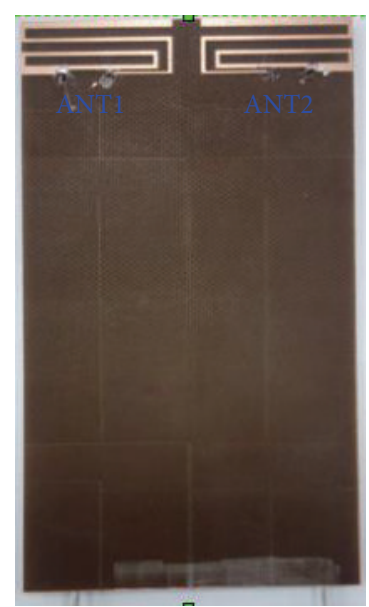

(a)

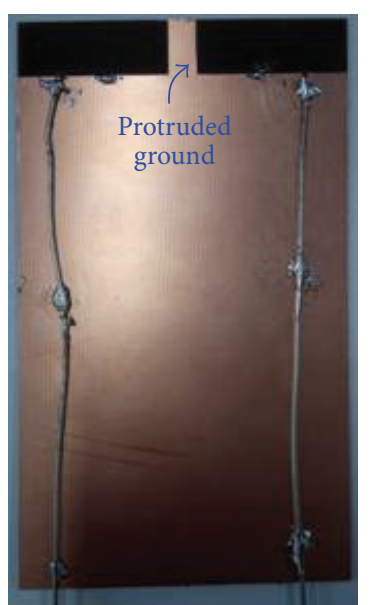

(b)
Figure 4: Manufactured planar antenna array for mobile phone applications.

the protruded ground plays an important role in the performances of the antenna array, and its simple structure is also desired in practical antenna array design for mobile phone applications.

\section{Results and Discussions}

The planar antenna array for mobile phone applications, as shown in Figure 4, was fabricated and tested. The simulations have been conducted by means of a commercial software based on the FEM technique (HFSS Version 13), while the measurements have been performed using an Agilent Vector Analyzer E8357A and a SATIMO Chamber. During the test, the plastic housing is also applied to simulate the real mobile phone circumstances. The experimental results and their corresponding discussions will be presented in separate subsections. 
TABLE 1: Diversity performances of the proposed antenna array.

\begin{tabular}{lccccccccc}
\hline Frequency (MHz) & 850 & 925 & 960 & 1750 & 1875 & 2000 & 2350 & 2550 & 2650 \\
ECC & 0.32 & 0.37 & 0.15 & 0.03 & 0.15 & 0.11 & 0.08 & 0.02 & 0.01 \\
MEG1 (dB) & -12.1 & -11.2 & -9.1 & -7.1 & -5.6 & -4.9 & -3.8 & -4.8 & -2.7 \\
MEG2 (dB) & -13.7 & -10.5 & -7.8 & -8.2 & -4.1 & -4.7 & -4.3 & -3.2 & -3.6 \\
\hline
\end{tabular}

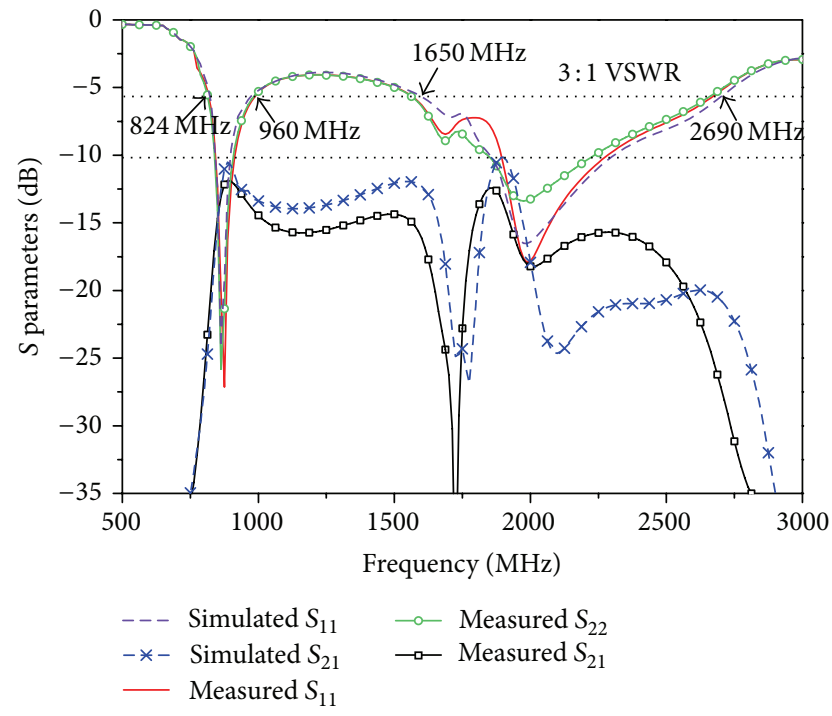

FIGURE 5: Simulated and measured $S$ parameters of the proposed antenna array.

3.1. S Parameters. The simulated and experimental $S$ parameters of the proposed antenna array are shown in Figure 5. It is seen that the curves of the measured results show the same trend with the simulated ones, while some variations are mainly due to the manufacture tolerance, especially for the upper band. Specially, based on the design specification of $3: 1$ VSWR, the frequency band of the proposed antenna array is $824 \sim 960 \mathrm{MHz}$ and $1650 \sim 2690 \mathrm{MHz}$, covering the seven operative bands of GSM850/900/1800/1900/ UMTS2100/LTE2300/2500 for WWAN/LTE mobile phone applications. The isolation between the antenna elements is above $10 \mathrm{~dB}$ over the whole band, and this can meet the requirements of MIMO mobile phones.

3.2. Radiation Patterns. The far-field measurements were carried out in a SATIMO Chamber and the obtained 2D radiation patterns are depicted in Figure 6. As the two array elements have identical dimensions and are symmetrically placed, their radiation pattern is almost the same. Therefore, to show clearly, the radiation pattern of only one antenna element is drawn at one picture. Figures 6(a) and 6(b) show the radiation patterns for $x o z, y o z$, and xoy planes at $900 \mathrm{MHz}$ for ANT1 and $2500 \mathrm{MHz}$ for ANT2, respectively. At $900 \mathrm{MHz}$ for ANT1, smooth variations are observed in the xoy plane, which means that the antenna elements are much dipole-like and are able to provide reliable coverage over the operative bands of GSM850/900. By contrast, in the xoy plane at $2500 \mathrm{MHz}$ for ANT2, the radiation pattern is not omnidirectional. Nevertheless, due to the almost equivalent power of the vertical and horizontal polarization from the base station, the $x o z$ and $y o z$ planes can compensate for this deficiency.

3.3. Efficiency and Peak Gain. To evaluate the radiation characteristics of the array elements, the efficiencies and peak gains are tested, as shown in Figures 7 and 8, respectively. It is observed that the antenna efficiencies of the two array elements are larger than $45 \%$, while the peak gains are in the range from $0.47 \mathrm{dBi}$ to $1.06 \mathrm{dBi}$ for the lower band. For the upper band, the measured antenna efficiencies and peak gains of the two array elements are both much larger, which are over $54.3 \%$ and $1.05 \mathrm{dBi}$, respectively. Specially, by comparing the two curves in Figures 7 and 8, it is noted that the efficiencies and peak gains of the two antenna elements agree well with each other. Accordingly, the obtained efficiencies and peak gains demonstrate that the proposed antenna array has good radiation characteristics and can meet the design requirements.

3.4. ECC and MEG. In order to evaluate the diversity performances of the antenna array, the envelop correlation coefficient (ECC) [12] and mean effective gain (MEG) [13], which are regarded as critical performance parameters to estimate the MIMO system, are calculated from the measured far-field data. From Table 1, it is found that the calculated ECCs are less than 0.37, meaning that the mutual coupling between the two antenna elements is acceptable [14]. At the same time, the MEGs can meet the requirements of |MEG1 MEG2 $<3 \mathrm{~dB}$ over the whole band, demonstrating that the antenna array has good power balance. Therefore, the proposed antenna array has excellent diversity performances and is suitable for MIMO systems.

3.5. Capacity. The channel capacity is another important parameter to estimate the MIMO system. In this paper, the number of transmitting and receiving antenna elements both is two, and the designed antenna array is assigned on the receive side, while the correlation on the transmit side is zero. Assuming that the channel is Rayleigh fading channel, then the ergodic capacity can be achieved by means of the measured efficiency and ECCs [15]. Figure 9 shows the calculated channel capacity, and it is obvious that, obtaining a certain channel capacity, the differences in SNR are mainly determined by the antenna efficiency because the correlation between the antenna elements is very low. 

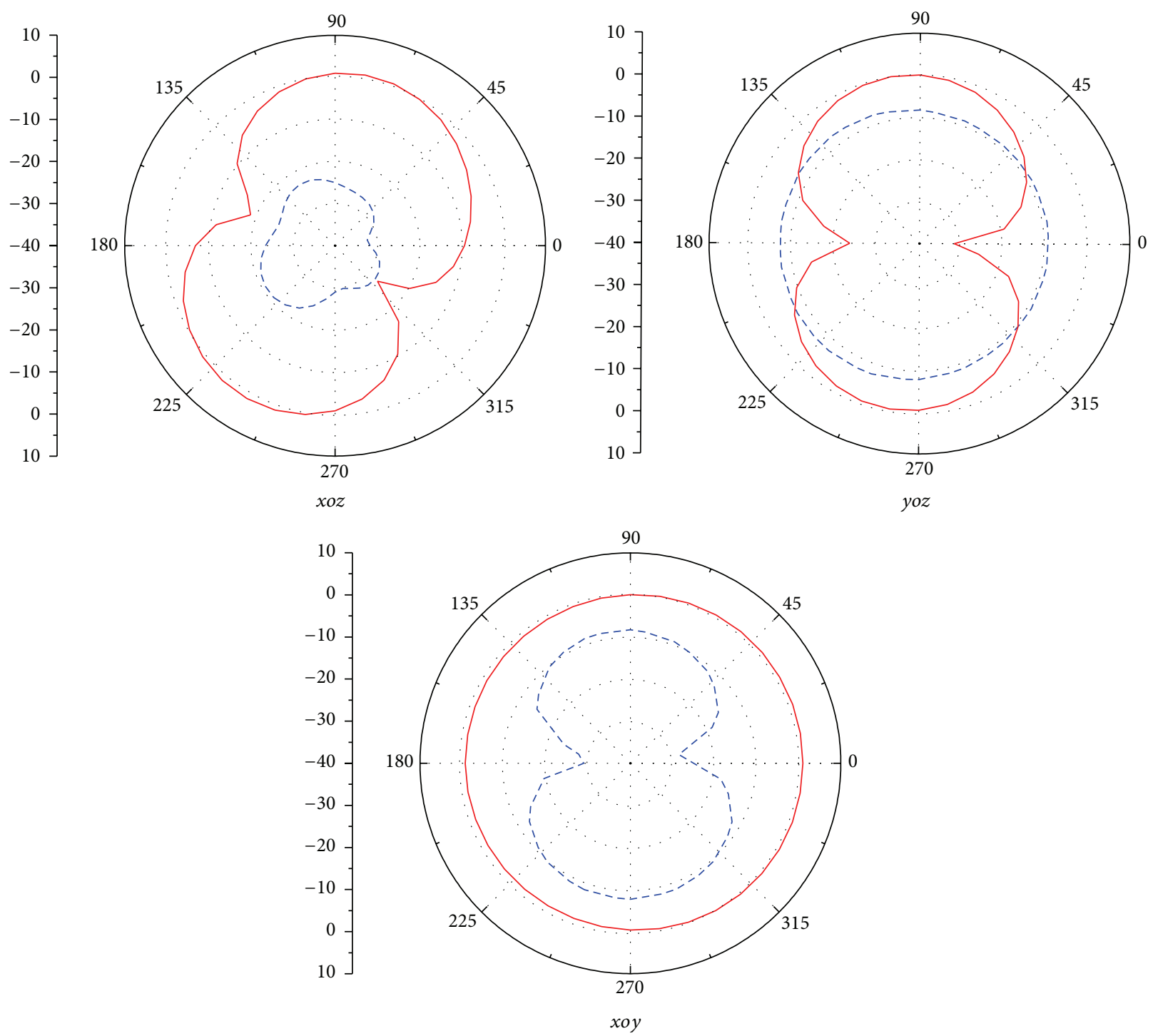

(a) $900 \mathrm{MHz}$
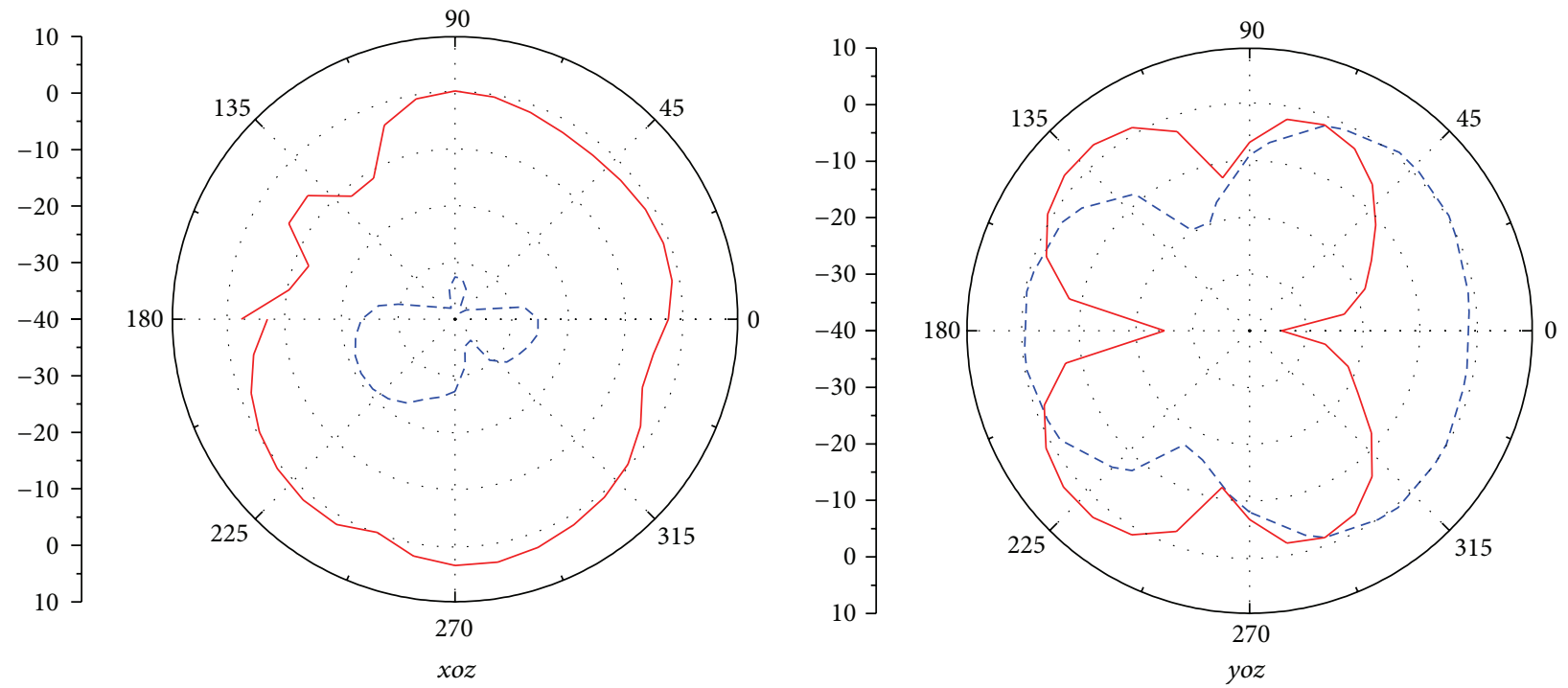

FIgUre 6: Continued. 


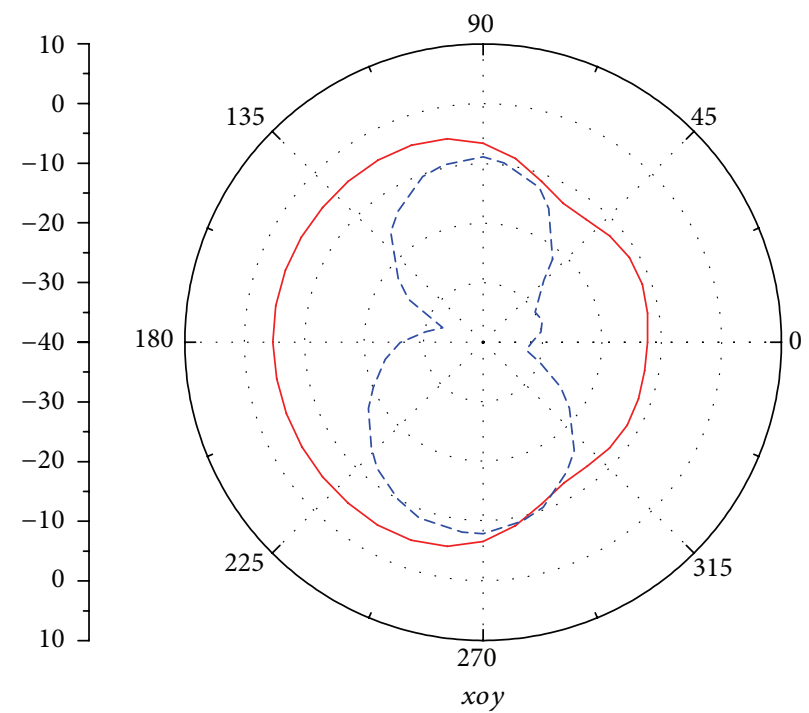

(b) $2500 \mathrm{MHz}$

FIGURE 6: Measured 2D radiation patterns of the antenna element at different frequencies. (a) $900 \mathrm{MHz}$ (ANT1); (b) 2500 MHz (ANT2) $\left(-E_{\theta} ;---E_{\varphi}\right.$, unit: $\left.\mathrm{dBi}\right)$.

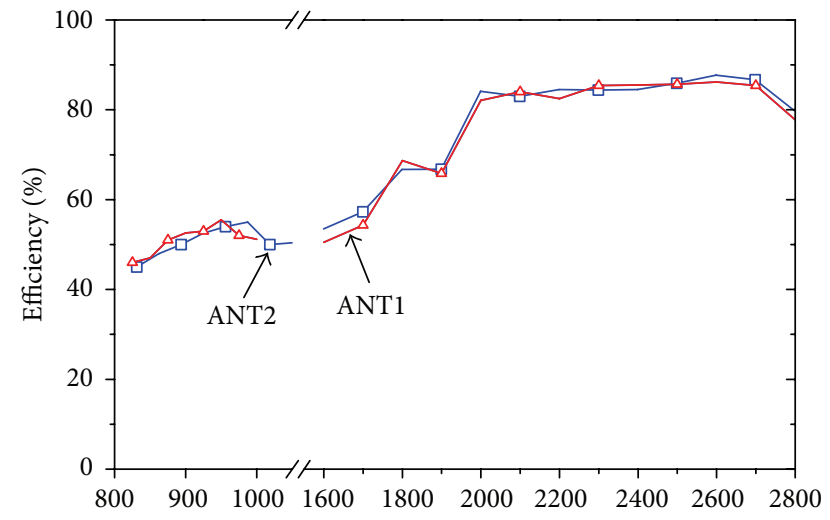

Figure 7: Measured efficiencies of the two array elements.

\section{Conclusion}

A printed antenna array for seven-band MIMO mobile phone applications has been presented and studied. Each of the array elements is composed by a driven strip and a wandering shorting strip. With the effects of the protruded ground, the antenna array is able to cover the frequency bands of 824 $960 \mathrm{MHz}$ and $1650 \sim 2690 \mathrm{MHz}$, with good isolation of more than $10 \mathrm{~dB}$. The experimental and calculated results infer that the proposed antenna array has good radiation characteristics and diversity performances over the whole operative bands. In addition, due to the planar and simple antenna structure, the proposed antenna array is quite suitable for practical MIMO mobile phone applications.

\section{Conflict of Interests}

The authors declare that there is no conflict of interests regarding the publication of this paper.

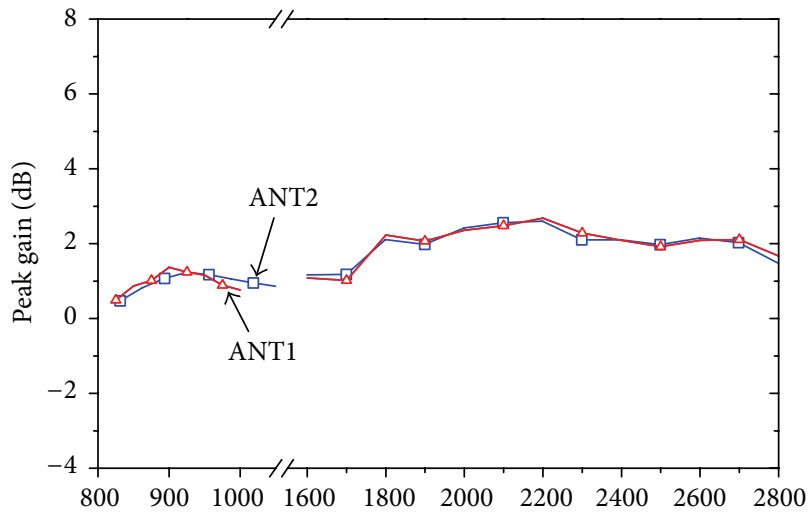

FIGURE 8: Measured peak gains of the two array elements.

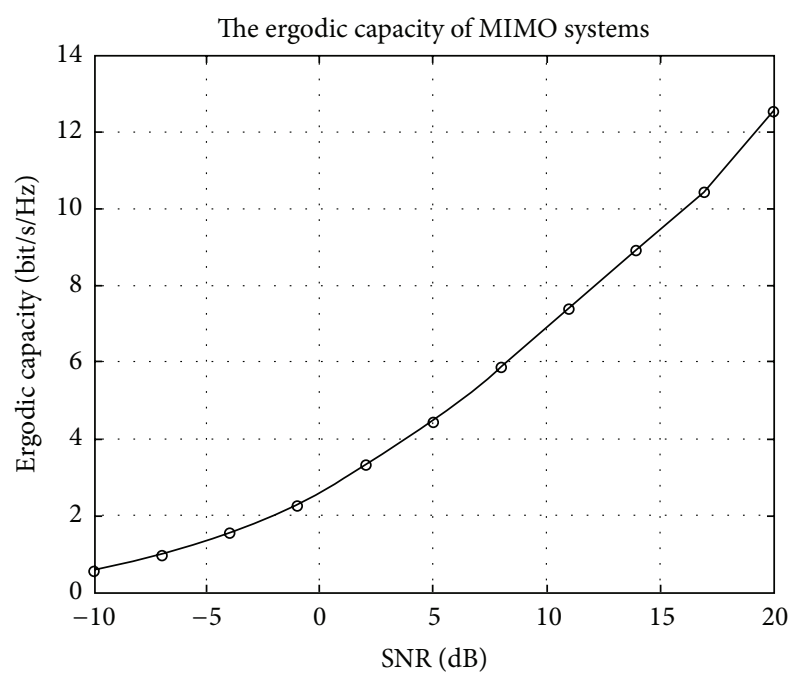

FIGURE 9: Calculated channel capacity as a function of SNR. 


\section{Acknowledgments}

This work is supported by the Youth Fund of Hebei North University (no. 120164), the Science and Technology Bureau of Zhangjiakou (no. 1321019B), and the Ministry of Science and Technology (no. 2012BAJ18B08).

\section{References}

[1] N. Prasad, H. Zhang, H. Zhu, and S. Rangarajan, "Multi-user MIMO scheduling in the fourth generation cellular uplink," IEEE Transactions on Wireless Communications, vol. 12, no. 9, pp. 4272-4285, 2013.

[2] L. Juho and H. Jin-Kyu, "MIMO technologies in 3GPP LTE and LTE-advanced," EURASIP Journal on Wireless Communications and Networking, vol. 2009, Article ID 302092, 2009.

[3] Q. H. Li, G. J. Li, W. Lee et al., "MIMO techniques in WiMAX and LTE: a feature overview," IEEE Communications Magazine, vol. 48 , no. 5, pp. 86-92, 2010.

[4] T. Kanesan, S. Rajbhandari, E. Giacoumidis, and I. Aldaya, "Nonlinear limit of alternative method to $2 \times 2$ MIMO for LTE RoF system," Electronic Letters, vol. 50, no. 4, pp. 300-301, 2014.

[5] K.-L. Wong, T.-W. Kang, and M.-F. Tu, "Internal mobile phone antenna array for LTE/WWAN and LTE MIMO operations," Microwave and Optical Technology Letters, vol. 53, no. 7, pp. 1569-1573, 2011.

[6] Y.-L. Ban, S. Yang, Z. Chen, K. Kang, and J. L.-W. Li, "Decoupled planar WWAN antennas with T-shaped protruded ground for smartphone applications," IEEE Antennas and Wireless Propagation Letters, vol. 13, pp. 483-486, 2014.

[7] J. U. R. Kazim, S. Ahmad, U. Ali, and O. Owais, "Design of a broadband planar monopole MIMO antenna," Microwave and Optical Technology Letters, vol. 56, no. 9, pp. 1994-1997, 2014.

[8] X. Y. Tang, K. Mouthaan, and J. C. Coetzee, “Tunable decoupling and matching network for diversity enhancement of closely spaced antennas," IEEE Antennas and Wireless Propagation Letters, vol. 11, pp. 268-271, 2012.

[9] M. Han and J. Choi, "MIMO antenna using a decoupling network for 4G USB dongle application," Microwave and Optical Technology Letters, vol. 52, no. 11, pp. 2551-2554, 2010.

[10] J. Oh and K. Sarabandi, "Compact, low profile, common aperture polarization, and pattern diversity antennas," IEEE Transactions on Antennas and Propagation, vol. 62, no. 2, pp. 569-576, 2014.

[11] S.-M. Wang, L.-T. Hwang, F.-S. Chang, and C.-F. Liu, "Design of high port isolation access point MIMO system using centrally located hexahedron," Electronics Letters, vol. 50, no. 4, pp. 248249, 2014.

[12] M. Pelosi, M. B. Knudsen, and G. F. Pedersen, "Multiple antenna systems with inherently decoupled radiators," IEEE Transactions on Antennas and Propagation, vol. 60, no. 2, pp. 503-515, 2012.

[13] A. A. Glazunov, A. F. Molisch, and F. Tufvesson, "Mean effective gain of antennas in a wireless channel," IET Microwaves, Antennas and Propagation, vol. 3, no. 2, pp. 214-227, 2009.

[14] X. Zhao and J. Choi, "Design of a MIMO antenna with low ECC for a $4 \mathrm{G}$ mobile terminal," Microwave and Optical Technology Letters, vol. 56, no. 4, pp. 965-970, 2014.

[15] A. Jemmali, M. Torabi, and J. Conan, "Performance analysis of MIMO schemes in 3GPP long term evolution system," Wireless Personal Communications, vol. 82, no. 2, pp. 1107-1125, 2015. 

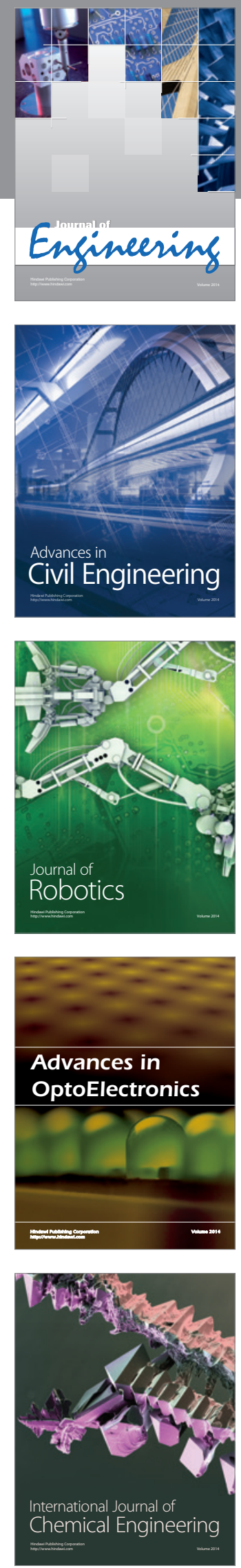

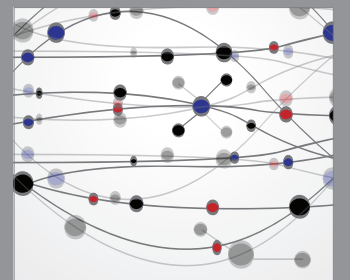

The Scientific World Journal
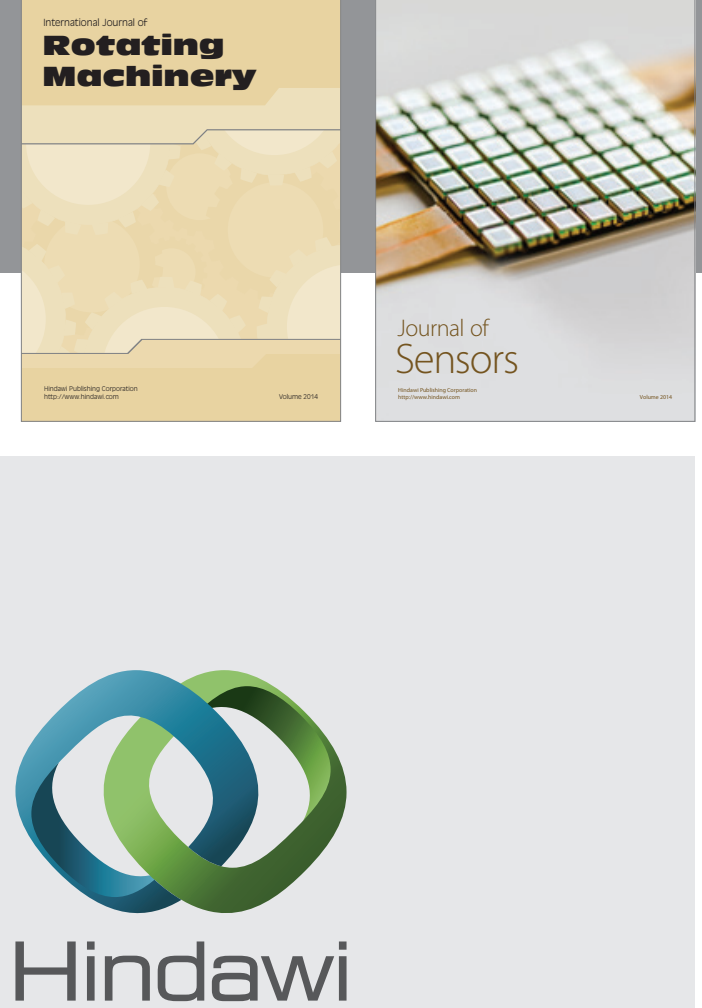

Submit your manuscripts at http://www.hindawi.com
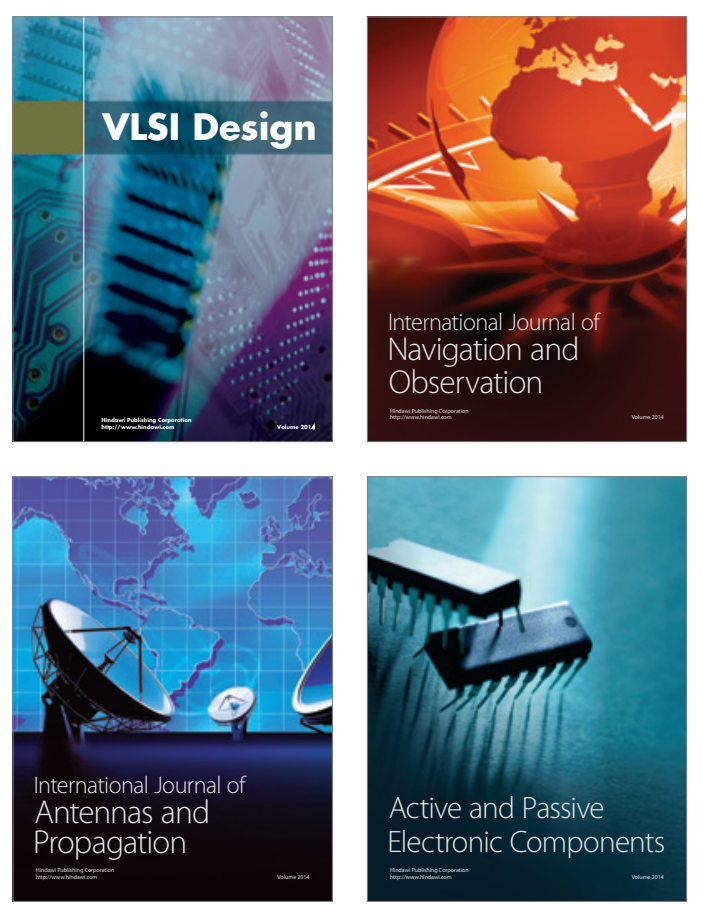
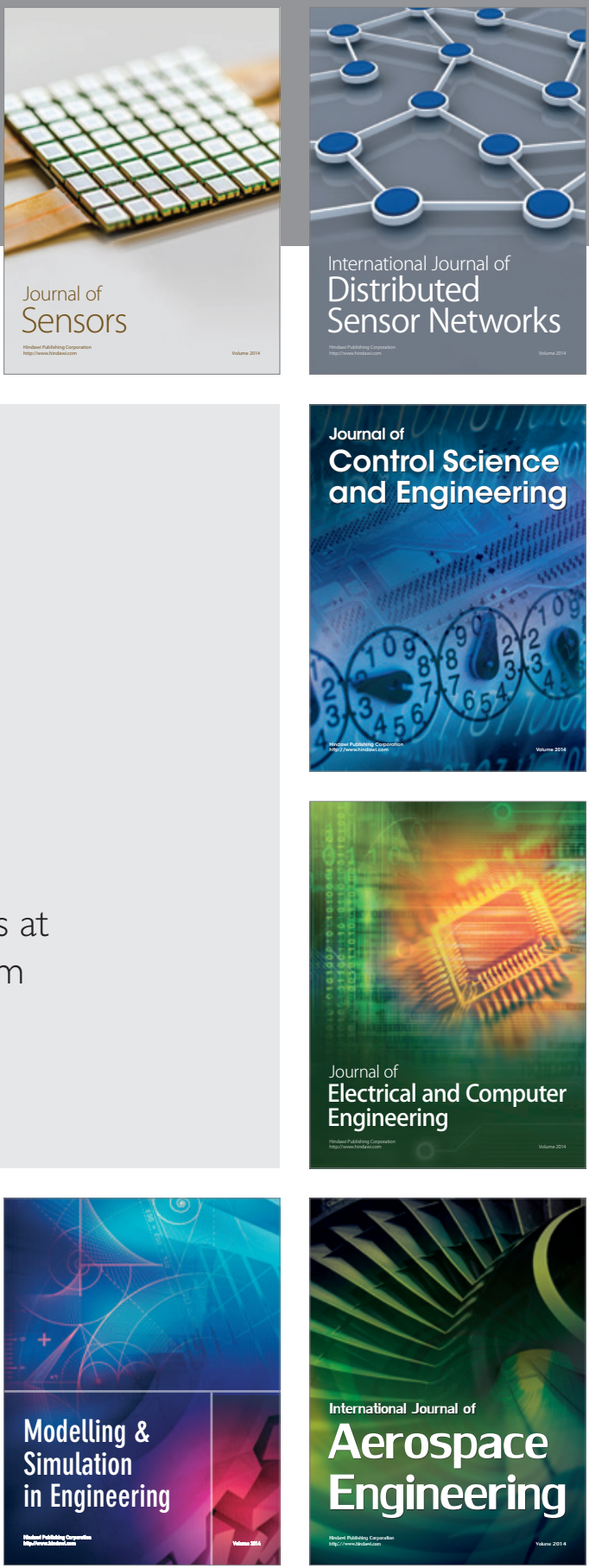

Journal of

Control Science

and Engineering
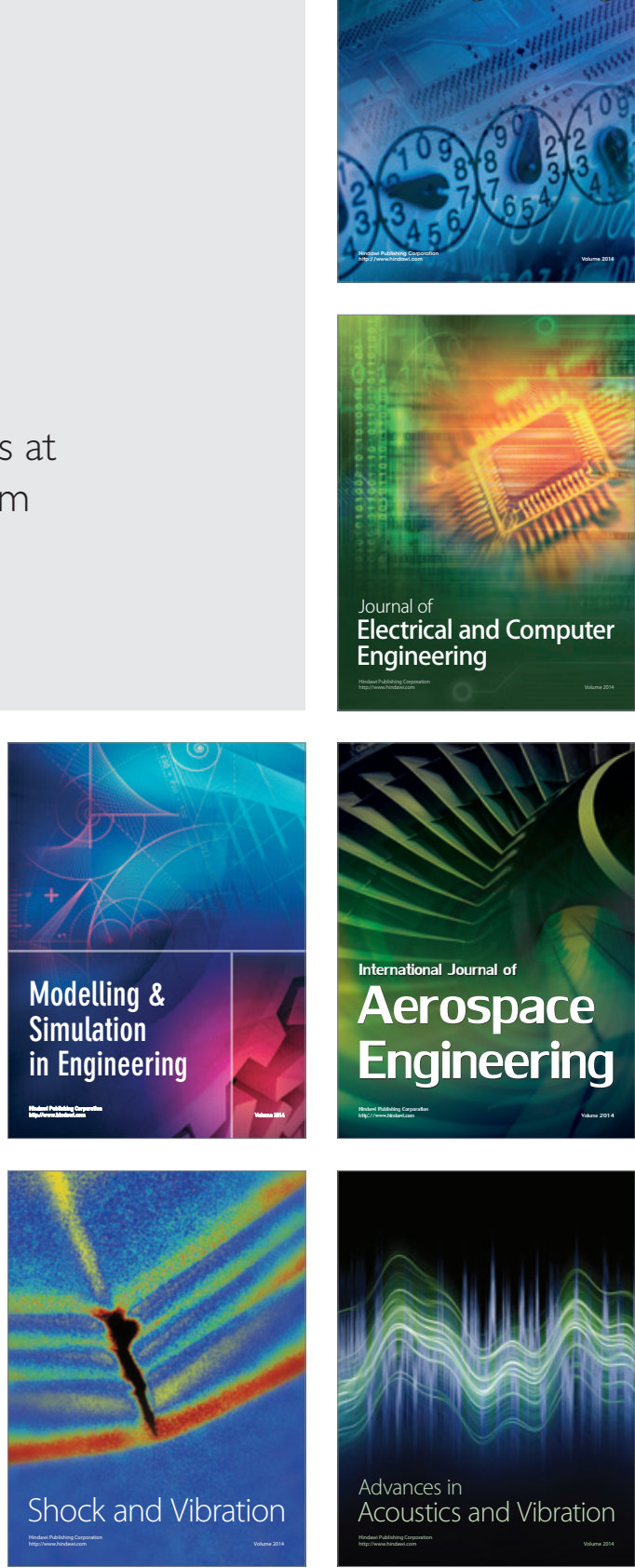